\title{
Influenza A Virus Mediated Downregulation of Progesterone Receptor Membrane Component-1 Inhibits RIG-I-dependent Antiviral Response in Central Nervous System
}

\section{Kun Huang}

Huazhong Agricultural University

Yufei Zhang

Huazhong Agricultural University

Wenxiao Gong

Huazhong Agriculture University

\section{Yong Yang}

Huazhong Agricultural University

Lili Jiang

Huazhong Agricultural University

Lianzhong Zhao

Huazhong Agricultural University

\section{Ying Yang}

Huazhong Agricultural University

\section{Yanming Wei}

Huazhong Agricultural University

Chengfei Li

Huazhong Agricultural University

\section{Xinglin He}

Huazhong Agricultural University

Xiaomei Sun

Huazhong Agricultural University

Zhong Zou

Huazhong Agricultural University

Meilin Jin ( $\square$ jml8328@126.com )

Huazhong Agricultural University: Huazhong Agriculture University https://orcid.org/0000-0003-43324083

\section{Research}


Keywords: H5N6 virus, CNS, PGRMC1, RIG-I, Virus replication

Posted Date: October 15th, 2020

DOI: https://doi.org/10.21203/rs.3.rs-90902/v1

License: (c) (1) This work is licensed under a Creative Commons Attribution 4.0 International License. Read Full License 


\section{Abstract}

Background: The influenza A virus (IAV) enters the central nervous system (CNS) via multiple routes and causes neurological symptoms. In this process, it develops multiple strategies to escape the host antiviral immune system, and infects the central nervous system (CNS). Progesterone receptor membrane component-1 (PGRMC1) is highly expressed in the CNS, where it exerts a neurotrophic effect. However, how PGRMC1 affects IAV remains unclear.

Methods: In this study, we aimed to investigate the role of PGRMC1 in regulating antiviral defense response in brain tissue. Toward this, we used both mouse model of IAV infection and the human neuroblastoma cell line SK-N-SH and human brain glioma cell line U251. High-throughput RNA sequencing (RNA-seq) was used to obtain an unbiased profile of the cellular response to IAV H5N6 infection in mice brain.

Results: Here, RNA-seq revealed 240 differentially expressed genes in the IAV-infected brains. Among the significantly down-regulated genes, we focused on the gene encoding progesterone receptor membrane component-1 (PGRMC1) and observed that IAV H5N6 infection clearly inhibited PGRMC1 in both neuroblastoma and glioma cells. Furthermore, treatment with AG205, a PGRMC1-specific inhibitor, or PGRMC1 knockout promoted H5N6 multiplication in vitro, while overexpression of PGRMC1 resulted in opposite effects. Furthermore, AG205 treatment or PGRMC1 knockout significantly inhibited RIG-Imediated IFN- $\beta$ signaling pathway and reduced the levels of several antiviral proteins (Mx1 and ISG15). In addition, PGRMC1-mediated regulation of IFN signaling relied on inhibition of the expression and ubiquitination of RIG-I.

Conclusion: Conclusively, our results show for the first time that IAV H5N6 down-regulates PGRMC1 expression to contribute to virus proliferation by inhibiting RIG-I-mediated IFN- $\beta$ production in the brain. These findings may offer new insights regarding the interplay between IAV and host factors that may impact IAV pathogenicity in the brain.

\section{Background}

Influenza A virus (IAV), the causative agent of flu, causes acute respiratory distress syndrome among humans and many mammalian and avian species. Novel IAV have emerged via antigenic drift and reassortment events with other IAV. The highly pathogenic avian influenza virus H5N1 (HPAIV/H5N1) was first detected in a domestic goose in Guangdong province in China in 1996 [1]. Since then, HPAIV has repeatedly caused devastating outbreaks in wild birds and poultry, as well as sporadic human infections with high mortality. Since 2013, five H7N9 influenza epidemic waves have resulted in 1344 cases, with 511 deaths in China [2]. In addition, HPAIV/H5N6 has replaced H5N1 as one of the dominant IAV subtypes circulating in waterfowls and causing human infections in China [3]. Therefore, the continuous evolution of the virus represents a long-term threat to public health and the poultry industry. 
IAV requires the host cell machinery to support replication of its genome and for the production of new virions [4]. Conversely, host cells produce various factors that target different steps in the virus life cycle to restrict virus infection and multiplication. Being part of the first line of defense against infections, type I interferons (IFNs) are key components of the host antiviral innate immune response and are modulators of adaptive immune response. The innate immune response is triggered in an IAV-infected host to block virus replication and accelerate viral clearance. Host recognition of pathogens via pattern recognition receptors (PRRs) is the first step in triggering an immune response. The IAV RNA is recognized by various PRRs, including Toll-like receptors (TLR3, TLR7, TLR8) retinoic acid-inducible gene I (RIG-I), and NOD-like receptor (NLRP3) [5]. These sensors activate signaling cascades resulting in the expression of specific inflammatory cytokines and chemokines [6]. Cytokines play various roles, such as direct inhibition of viral replication and activation of the cytolytic functions of T cells, whereas chemokines recruit innate immune cells such as macrophages, neutrophils, natural killer (NK) cells, and inflammatory monocytes to the lungs and airways [7]. However, viruses have evolved sophisticated mechanisms to evade or counteract host innate immune responses. In particular, virus-encoded genes inhibit host innate antiviral responses by directly targeting IFN gene expression or/and IFN-induced host effector molecules. The non-structural protein 1 (NS1) is best characterized and the most important IFN antagonist protein of IAV. NS1 can block type I interferon signaling downstream of RIG-I or may block RIG-I ubiquitination [8]. PB1-F2 from IAV strain A/Puerto Rico/8/1934 can interact with mitochondrial antiviral signaling (MAVS) to inhibit interferon production [9].

The central nervous system (CNS) disease is one of the most common extra-respiratory tract complications of IAV infections [10,11]. IAV has been associated with CNS disease since the 1918-H1N1 pandemic, and CNS disease has been observed during all subsequent pandemics, as well as during seasonal epidemics, with occasional detection of IAV in the CNS of humans [12, 13]. Zoonotic IAV only sporadically infects humans, but when they do, they are frequently linked to severe and systemic disease. HPAIV H5N1 and H7N9 viruses, two recently identified zoonotic IAVs, are associated with CNS disease $[14,15]$. The HPAI H5N1 virus is possibly the most neurotropic IAV known and has frequently been associated with CNS disease in humans and in other naturally and experimentally infected mammalian species. IAV needs to infiltrate the CNS to infect, replicate, and spread throughout the CNS. IAV reaches the CNS via the olfactory, sympathetic, trigeminal, vagus, and possibly other cranial nerves $[16,17]$ However, there is still little knowledge on the IAV regulating host responses in brain.

Progesterone receptor membrane component-1 (PGRMC1) is a 26-28 kDa membrane-spanning protein with a short extracellular domain, a single transmembrane region, and a cytoplasmic domain. The cytoplasmic segment contains a cytochrome P450 b5/heme binding sequence, which can bind to various pharmacological compounds [18]. PGRMC1, also called Dap1, human progesterone receptor (HPR6.6), and IZA, is a membrane-associated progestin receptor (MAPR) occurring in different species [19]. It is highly expressed in the CNS, where it exerts a neurotrophic effect [20]. PGRMC1 has been shown to be involved in multiple cellular functions such as cholesterol regulation, axonal guidance, endocytosis, and alteration of reproductive behaviors [21-24] However, how PGRMC1 affects pathogens, especially IAV, remains unclear. 
In this study, we aimed to investigate the role of PGRMC1 in regulating antiviral defense response in brain tissue. Toward this, we used both mouse model of IAV infection and human neuroblastoma and glioma cell lines. High-throughput RNA sequencing (RNA-seq) was used to obtain an unbiased profile of the cellular response to IAV H5N6 infection in mice brain. We demonstrated that down-regulation of PGRMC1 is required for IAV replication and propagation in both the human neuroblastoma cell line SK-N-SH and human brain glioma cell line U251. Furthermore, we showed that the PGRMC1-specific inhibitor, AG205, or PGRMC1 knockout, functions as IFN antagonist in IAV infection by negatively regulating type I IFN induction and antiviral gene expression. Our findings reveal a novel mechanism via which IAV H5N6 inhibits host IFN response and emphasizes the vital role of PGRMC1 in modulating antiviral defenses in the brain.

\section{Methods}

\section{Cells and viruses}

The human neuroblastoma cell line, SK-N-SH, human brain glioma cell line, U251, and the Madin-Darby canine kidney cell line (MDCK), were cultured in Dulbecco's modified Eagle's medium (Hyclone, Logan, UT, USA) supplemented with $10 \%$ fetal bovine serum (PAN Biotech) and $100 \mathrm{U} / \mathrm{mL}$ penicillin-streptomycin (Thermo Fisher Scientific, Inc.). HEK293T cells were cultured in Roswell Park memorial Institute (RPMI)-1640 medium (Invitrogen).

The IAV strain used in this study was the A/duck/Hubei/WH18/2015(H5N6) (JX) (Genebank assession number: KX652135) strain isolated from infected duck lung in our laboratory. H5N6 was grown in 9-dayold embryonated eggs. The titers of IAV in culture media were determined using the $50 \%$ tissue culture infective dose (TCID50) and plaque assays in MDCK cells [25].

\section{Mouse infection}

Female BALB/c mice aged 4-6 weeks were purchased from the Center for Animal Disease Control, Hubei Province. Mice $(n=11)$ were intranasally $(I N)$ infected with $50 \mu \mathrm{L}$ of $10^{5} \operatorname{TCID}_{50}$. The infected mice were observed daily for the signs of disease and death for 14 days post infection (dpi), while naive control mice were inoculated with phosphate buffered saline (PBS). Six mice in each group were sacrificed on 3 $\mathrm{dpi}$, and their brains were collected for further study. For pathogenicity analysis, three brains was split in half respectively. The right hemisphere was collected in $1 \mathrm{~mL}$ PBS supplemented with antibiotics (100 $\mathrm{U} / \mathrm{mL}$ penicillin-streptomycin) and the left hemisphere was placed in formalin. For transcriptomic analysis, three other brains were harvested and stored in TRIzol reagent (Invitrogen, Carlsbad, CA, USA).

\section{Immunohistochemistry (IHC)}

The fixed brains were paraffin-embedded, sectioned, and stained with a rabbit-anti-NP specific monoclonal antibody (GeneTex). Goat anti-rabbit immunoglobulin conjugated to peroxidase (Maxim Bio, 
Fujian, China) was used as the secondary antibody. The sections were screened using an Olympus BX51 microscope coupled to a camera.

\section{Plasmid construction}

The IFN- $\beta$-luc, RL-TK, RIG-I, MDA-5, TBK-1, IKKE, IFN regulatory factor (IRF3), IRF3-5D, and pUb-HA plasmids were kindly provided by Prof. Zhengfan Jiang (Peking University) [4]. PGRMC1 was cloned in the p3.Flag-CMV-14 vector. A lentiviral vector encoding EGFP-Cas9, the packaging plasmid pMD2.G, and the envelope plasmid psPAX2 were kindly provided by Prof. Lisheng Zhang (Huazhong Agricultural University). The PGRMC1 guide RNA was cloned in the lentiviral vector. Plasmids expressing Flag- RIG-I were amplified from HA-RIG-I and cloned into p3FLAG-CMV-14 vectors.

\section{RNA sequencing and gene expression analysis}

Total RNA was isolated and purified using the TRIzol reagent (Invitrogen, Carlsbad, CA, USA) following the manufacturer's procedure and was dispatched to LC-Bio Corporation (Hangzhou, Zhejiang, China) for microarray analysis. The differentially expressed mRNAs and genes were selected using log2 (fold change) $>1$ or log2 (fold change) $<-1$ as cut-off. $P<0.05$ was considered statistically significant $(R$ package Ballgown).

\section{RNA extraction and quantitative reverse transcription- polymerase chain reaction (qRT-PCR)}

Total RNA was extracted from brains or cells using Trizol according to the manufacturer's instructions. Next, the mixed genomic DNA was digested with DNase I and $1 \mu \mathrm{g}$ RNA was reverse transcribed using the reverse transcriptase (TaKaRa Biotechnology, Dalian, China) from avian myeloblastosis virus with oligo (dT) 18. cDNA $(0.5 \mu \mathrm{L})$ was used as a template for qRT-PCR. In addition, the reaction mixtures contained $5 \mu \mathrm{L} 2 \times$ SYBR Green master mix (Roche, Indianapolis, IN, USA), $0.25 \mu \mathrm{L}$ of each primer (10 mM), and $4 \mu \mathrm{L}$ ultrapure water. The assay was run on an ABIViiA 7 PCR system (Applied Biosystems, Waltham, MA, USA). The expression of each gene was normalized to that of glyceraldehyde 3-phosphate dehydrogenase (GAPDH) as a control.

\section{Luciferase reporter assays}

HEK293T cells cultured in 24-well plates were incubated with the PGRMC1 inhibitor AG205 (SigmaAldrich) or dimethyl sulfoxide (DMSO) for $24 \mathrm{~h}$. The cells were transfected with IFN- $\beta$-luc (200 ng/well) and the internal control pRL-TK (10 ng/well). After $24 \mathrm{~h}$, the cells were transfected with poly(I:C) $(200 \mathrm{ng} / \mathrm{mL}$ ) and incubated for $12 \mathrm{~h}$, followed by analysis of cell lysates for luciferase activity with a dual luciferase reporter assay kit (Promega) according to the manufacturer's instructions. HEK293T cells seeded in 24-well plates were co-transfected with the expression plasmid harboring PGRMC1 or the empty vector, IFN- $\beta$-luc (200 ng/well), and the internal control pRL-TK (10 ng/well), together with the adaptor molecules(RIG-I, MDA-5, TBK-1, IKKE, IRF3, IRF3-5D) of each signaling pathway. Luciferase activity was measured as mentioned above $24 \mathrm{~h}$ post-transfection. 


\section{Western blotting}

The cells were washed with cold PBS and lysed on ice in Tris lysis buffer (Cell Signaling Technology, Danvers, MA, USA) containing 1\% EDTA-free protease inhibitors (Roche). To remove cell debris, the cell lysates were centrifuged at $12,000 \mathrm{rpm}$ for $15 \mathrm{~min}$ at $4^{\circ} \mathrm{C}$. The supernatant was resuspended in $1 \mathrm{x}$ sodium dodecyl sulfate (SDS) loading buffer, was resolved using SDS-polyacrylamide gel electrophoresis (SDS-PAGE), and transferred to nitrocellulose membranes (GE). The membrane was first probed using certain primary antibodies and then with horseradish peroxidase-conjugated goat anti-mouse or antirabbit secondary antibodies (Abcam; Cambridge, MA). Finally, a chemiluminescence imaging system (DNR, Neve Yamin, Israel) was used for the analysis and images were acquired.

\section{Generation of PGRMC1 knockout U251 cells}

PGRMC1-KO U251 cells were generated using the clustered regularly interspaced short palindromic repeats (CRISPR)/Cas9 system and lentiviral vectors as described previously [26]. The single guide RNA sequence targeting the human PGRMC1 (5'-GCTCTACAAGATCGTGCGCG-3') was cloned in the BsmBI site of the EGFP-Cas9 lentiviral vector. The EGFP-Cas9 lentiviral vector, psPAX2, and pMD2.G were cotransfected in $293 \mathrm{~T}$ cells. The cell supernatant was harvest $48 \mathrm{~h}$ post-transfection. Then, U251 cells were incubated with the viral supernatant or the empty vector lentivirus. Finally, the monoclonal cells were sorted using flow cytometry and seeded in a 96-well plate to generate clonal cell lines. PGRMC1 knockout was confirmed via sequencing and western blotting.

\section{Co-immunoprecipitation (co-IP) assay}

Wild type (WT) or PGRMC1-KO U251 cells were transfected with pCAGGS-HA-Ub together with p3.FlagRIG-I. At $48 \mathrm{~h}$ post-transfection, the cells were lysed with buffer for western blotting and IP (Beyotime, Beijing, China) at $4^{\circ} \mathrm{C}$ for $30 \mathrm{~min}$. The supernatants were collected and incubated overnight with anti-HA magnetic beads (Bimake, Beijing, China) at $4^{\circ} \mathrm{C}$. Then, the beads were washed thrice with lysis buffer and eluted with $1 \times$ SDS loading buffer. Samples were tested using western blotting.

\section{Laboratory facility}

All experiments involving live viruses were performed in a biosafety level 3 (BSL3) facility in accordance with the institutional biosafety manual. The animals were housed in negative-pressure isolators with high efficiency particulate air filter in the BSL3 facility.

\section{Statistical analyses}

The results are expressed as means \pm standard deviations (SD). Data were collected from triplicate experiments and are representative of at least three independent experiments. Groups were compared using one-way analysis of variance (ANOVA) of GraphPad Prism (San Diego, CA, USA). The level of significance was set at $p<0.05$. Two-way ANOVA was used when appropriate. Mice survival were compared using the Mantel-Cox version of the log-rank test. 


\section{Results}

\section{Infection of BALB/c mice with the JX H5N6 virus}

Previously, we have demonstrated that HPAIV H5N6 (A/duck/Hubei/WH18/2015, abbreviated as JX) is neurotoxic and highly pathogenic to mice. To establish animal models of brain damage caused by IAV, $\mathrm{BALB} / \mathrm{c}$ mice were inoculated intranasally with $10^{5} \mathrm{TCID}_{50} / 50 \mu \mathrm{L}$ inoculum/mouse of IAV JX, and the mock-treated mice were used as control. All mice infected with JX showed clearly detectable symptoms of the flu at $3 \mathrm{dpi}$, and showed drastic weight loss (Fig. 1A, F $(1,98)=293.2, \mathrm{P}<0.0001$ ). Strikingly, these symptoms had progressed to neurological symptoms such as balancing problems, hind limb weakness, and paralysis by 5 and $6 \mathrm{dpi}$ and mice began to die by $7 \mathrm{dpi}$. At the end of the experimental period, only two out of five mice survived (Fig. 1B, Mantel-Cox log-rank test, $P=0.0511$ ). Next, $I H C$ was performed to investigate whether the brain was successfully infected by the JX virus. Results suggested that the brains of mice were successfully infected (Fig. 1C, 1D). Meanwhile,IHC staining for IAV viral antigen revealed virus-positive neurons in the brains of mice sacrificed on $3 \mathrm{dpi}$. Virus antigen was detected in multiple areas of the brain, including cerebral cortex, brainstem, occipital lobe. For representative regions, an image of the cerebral cortex with a single staining of IAV antigen is shown (Fig. 1C). Brain tissue injury caused by JX virus involves sub-meningeal hemorrhage, degeneration, and necrosis of neurons and local glial cell nodules(Fig. 1D). Collectively, these results demonstrated that the JX virus can infect, replicate, and spread throughout the CNS, thereby successfully establishing a mouse model for further functional studies.

\section{Brain transcription profile after JX virus infection}

To determine the differential response of the host brain tissue to infection with JX and mock infection, RNA samples were prepared from whole brains and the complete gene expression profile was compared after whole transcriptome sequencing (Fig. 2). Histopathological and immunohistochemical examinations showed neuron necrosis, proliferation of glial cells, and viral antigen positivity on $3 \mathrm{dpi}$. Therefore, brain samples were obtained on this day. A cut off of $>2.0$ in fold change and $P<0.05$ was used initially to examine the significantly differentially expressed genes that were up- or down-regulated following JX infection (Fig. 2). In total, 32,716 genes were detected in the brain, 240 of which were identified as differentially expressed mRNAs between the virus-infected and uninfected mice. Among these, 179 genes were up-regulated with log2 fold change values ranging from 1.01 to 5.08 , while 61 genes were down-regulated with log2 fold change values ranging from 1 to 4.59 . The top 10 up-regulated genes listed were $C x x 1 b / C x x 1 a$, Scrt1, Bmyc, Pcp2, Car8,Arl4c, Fat2, Mybpc3, Rn7sk, and Irf2bp1 (Table 1). The top 10 most down-regulated genes were Nudc-ps1, Glrx5, Pgrmc1, Rnf113a2, Irgq, Rgs4, Tmem97, Zik1, Zcchc3, and Gng4 (Table 2). To further assess the connection between gene expression pattern and IAV infection-induced biological processes in the brain, functional classification of mRNA transcripts and pathway analysis were performed. These analyses revealed that these genes were involved in regulating neuronal differentiation, ion transmembrane transport, and protein binding. Some of the significant pathways involving the differentially expressed genes were associated with herpes 
simplex infection, glutamatergic synapse, and chemokine signaling pathway. Among the significantly down-regulated genes, we focused on PGRMC1 to evaluate their role in IAV infection in the brain.

Table 1

The top 10 most up-regulated genes of virus-infected mice compared to control mice

\begin{tabular}{|llll|}
\hline Symbol & Gene name & Log $_{2}$ FC & p-value \\
\hline Cxx1b;Cxx1a & CAAX box 1A ;CAAX box 1B & 5.68 & 0.014 \\
\hline Scrt1 & scratch family zinc finger 1 & 4.47 & 0.021 \\
\hline Bmyc & brain expressed myelocytomatosis oncogene & 4.35 & 0.046 \\
\hline Pcp2 & Purkinje cell protein 2 (L7) & 4.31 & 0.037 \\
\hline Car8 & carbonic anhydrase 8 & 4.25 & 0.044 \\
\hline Arl4c & ADP-ribosylation factor-like 4C & 3.94 & 0.046 \\
\hline Fat2 & FAT atypical cadherin 2 & 3.56 & 0.045 \\
\hline Mybpc3 & myosin binding protein C, cardiac & 3.51 & 0.038 \\
\hline Rn7sk & RNA, 7SK, nuclear & 3.32 & 0.05 \\
\hline Irf2bp1 & interferon regulatory factor 2 binding protein 1 & 3.27 & 0.046 \\
\hline
\end{tabular}

Table 2

The top 10 most down-regulated genes of virus-infected mice compared to that in the control mice

\begin{tabular}{|llll|}
\hline Symbol & Gene name & Log $_{2}$ FC & p-value \\
\hline Nudc-ps1 & nuclear distribution gene C homolog (Aspergillus), pseudogene 1 & 4.59 & 0.001 \\
\hline Glrx5 & glutaredoxin 5 & 3.87 & 0.043 \\
\hline Pgrmc1 & progesterone receptor membrane component 1 & 3.35 & 0 \\
\hline Rnf113a2 & ring finger protein 113A2 & 3.1 & 0.038 \\
\hline Irgq & immunity-related GTPase family, Q & 2.92 & 0.014 \\
\hline Rgs4 & regulator of G-protein signaling 4 & 2.6 & 0.007 \\
\hline Tmem97 & transmembrane protein 97 & 2.38 & 0.031 \\
\hline Zik1 & zinc finger protein interacting with K protein 1 & 2.16 & 0.034 \\
\hline Zcchc3 & zin(Kimura et al., 2006)c finger, CCHC domain containing 3 & 2.11 & 0.019 \\
\hline Gng4 & guanine nucleotide binding protein (G protein), gamma 4 & 2.1 & 0.014 \\
\hline
\end{tabular}




\section{H5N6 down-regulated the expression of PGRMC1 in vitro}

It is well-known that the nervous tissue is composed of two primary cell types: neurons and glial cells. Neurons transmit nerve messages, while the surrounding glial cells are in direct contact with neurons (Kimura et al., 2006). To verify whether the H5N6 virus can down-regulate PGRMC1 in vitro, both human neuroblastoma cell line SK-N-SH and human glioma cell line U251 were used. SK-N-SH or U251 cells were infected with JX at multiplicity of infection (MOI) of 0.01 . At 24 and $48 \mathrm{hpi}, \mathrm{PGRMC1} \mathrm{mRNA}$ and protein levels were assessed using qRT-PCR and western blotting, respectively (Fig. 3). Compared to the mock, JX significantly inhibited the production of PGRMC1 mRNA in SK-N-SH after $24 \mathrm{~h}$, which was in agreement with the protein level (Fig. 3A, F $(1,23)=201.3, P<0.0001,3 C)$. At $48 \mathrm{hpi}$, the extent of PGRMC1 protein inhibition by the JX virus was lower than that at $24 \mathrm{hpi}$. Similar result was observed in U251 cells (Fig. 3B, $F(1,20)=87.57, P<0.0001,3 D)$. These results indicated that IAV JX may significantly inhibit the expression of PGRMC1 in agreement with those identified using RNA-seq.

\section{PGRMC1 inhibited H5N6 replication}

As IAV JX inhibited PGRMC1 expression, we speculated that PGRMC1 might participate in IAV JX replication. To better understand the biological role of PGRMC1 in H5N6 replication, AG205, a putative PGRMC1-specific inhibitor (Teakel et al., 2020), was used. SK-N-SH or U251 cells were seeded in 12-well plates and grown to $80 \%$ confluence. Then, the cells were treated with $15 \mu \mathrm{M}$ AG-205 or vehicle (cell culture grade DMSO) for $24 \mathrm{~h}$. Cells were infected with JX virus at MOI of 0.01. Viral NP mRNA levels and viral titers were evaluated at different time points (Fig. 4). NP mRNA levels in the two cell lines significantly increased in the AG205 group compared to that in the control (Fig. 4A, F $(1,21)=116.0, P<$ $0.0001 ; 4 B, F(1,19)=62.26, P<0.0001)$. Next, we observed that $A G 205$ significantly increased the viral titers in U251 $12 \mathrm{~h}, 24 \mathrm{~h}$ and $36 \mathrm{~h}$ post-infection (Fig. 4C, $\mathrm{F}(1,12)=44.47, \mathrm{P}<0.0001 ; 4 \mathrm{D}, \mathrm{F}(1,12)=37.78$, $\mathrm{P}<0.0001)$. However, viral titers significantly increased after AG205 treatment of SK-N-SH cells at $36 \mathrm{~h}$, although no effect on virus replication was evident $24 \mathrm{~h}$ after AG205 treatment. Subsequently, we compared the effect of AG205 on viral proliferation in the two cell types and observed that AG205 consistently enhanced the viral titers of IAV JX in the U251 cells compared to that in the SK-N-SH cells. The effect of PGRMC1 overexpression on viral replication was also determined by transfecting U251 cells with Flag-PGRMC1. The efficiency of PGRMC1 overexpression was determined using western blotting (Fig. 4F). As observed in the $\mathrm{TCID}_{50}$ assays, virus titer at 36 h.p.i were lower in the PGRMC1overexpressing groups than in the control group (Fig. 4E, $F(1,8)=26.36, P=0.0009)$. Taken together, these findings indicated that PGRMC1 was an IAV JX restriction factor.

\section{AG205 inhibited the IAV-induced RIG-I-dependent antiviral response}

AG205 affects virus multiplication more in U251 cells than in SK-N-SH cells. Consistently, the innate immune surveillance is mainly coordinated by glial cells in the CNS [27]. These CNS resident cells are assumed to orchestrate the immune response, which assists in combating infections in the brain. Hence, we hypothesized that AG205 may inhibit IAV H5N6-mediated innate immune response. To validate this 
hypothesis, we evaluated a series of immunological molecules in IAV JX-infected U251 cells, which were incubated with AG205 or DMSO (Fig. 5, Fig. 6). As expected, AG205 significantly down-regulated IFN- $\beta$ induced by IAV JX (Fig. 5A, F $(1,8)=126.1, \mathrm{P}<0.0001)$ ). In addition, the expression of the downstream effectors of IFN- $\beta$, namely, MX1 and ISG15, was suppressed (Fig. 6). We further observed that AG205 markedly inhibited poly (I:C)-mediated IFN- $\beta$ induction (Fig. 5B-G). The molecules upstream of IFN- $\beta$, such as RIG-I, TBK1, and IRF3, were inhibited by AG205. MX1 and ISG15 were also suppressed. Overall, these observations suggested that AG205 inhibited the type I interferon response.

\section{AG205 inhibited RIG-I-mediated IFN- $\beta$ production}

The RIG-I signaling pathway is essential for IAV recognition. Once activated, RIG-I/MAVS induces the activation of transcription factor IRF 3 and NF-KB, ultimately resulting in establishment of host IFNmediated antiviral response (Iwasaki and Pillai, 2014). To investigate how the PGRMC1 inhibitor AG205 regulates IFN- $\beta$, the IFN- $\beta$ signaling pathway was analyzed using IFN- $\beta$ luciferase activity assay (Fig. 7). AG205 or DMSO-pretreated 293 cells were co-transfected with the signal molecule expression plasmid, IFN- $\beta$ promoter luciferase reporter plasmid, and internal control pRL-TK. AG205 markedly inhibited RIG-Imediated IFN- $\beta$ production (Fig. 7A, $F(1,8)=156.1, \mathrm{P}<0.0001$ ). Interestingly, it did not affect other molecules (MDA5, $F(1,8)=3.410, P=0.1020 ;$ TBK1, $F(1,8)=0.08015, P=0.7843$; IKK- $\xi, F(1,8)=1.774$, $P=0.2196, I R F 3, F(1,7)=3.168, P=0.1183$; IRF3-5D, $F(1,8)=3.705, P=0.0905)$ that stimulate IFN- $\beta$ promoter luciferase activity (Fig. 6B-F). Notably, as shown in Fig. 7H (one-way ANOVA, F = 46.24, P = 0.005), we observed a dose-dependent relationship between AG205 and IFN- $\beta$ promoter luciferase activity. On the contrary, overexpression of PGRMC1 increased RIG-I-stimulated IFN- $\beta$ luciferase activity in a dose-dependent manner (Fig. 7G, one-way ANOVA, $F=78.66, P<0.0001$ ). Consequently, we concluded that down-regulation or functional inhibition of PGRMC1 reduces the ability of RIG-I to induce IFN- $\beta$ production in cells infected with IAV H5N6.

\section{PGRMC1 knockout promoted IAV replication by inhibiting the RIG-I-mediated IFN- $\beta$ signaling pathway}

As the PGRMC1 inhibitor AG205 has been shown to significantly promote IAV replication, we speculated whether PGRMC1 knockdown similarly affected the proliferation of IAV H5N6 in U251 cells. Toward this, PGRMC1 was knocked out using the lentiviral CRISPR-Cas9 system containing a pair of suitable guide RNAs driven by two independent U6 promoters on the same plasmid ( Fig. 8). After CRISPR/Cas9 gene editing and isolation of individual clones, PGRMC1 expression was analyzed using western blot analysis. Our results suggested that CRISPR/Cas9 targeting resulted in complete loss of the PGRMC1 protein (Fig. 8A). We first evaluated the effect of PGRMC1 deficiency on IAV JX propagation using a $\mathrm{TCID}_{50}$ assay. Indeed, cells with $P G R M C 1$ knockout produced more infectious viral particles than control U251 cells (Fig. 8B, $F(1,12)=70.53, P<0.0001)$, in agreement with the results obtained in cells treated with AG205. Furthermore, the expression of RIG-I (protein level, Fig. 6) and IFN- $\beta$ (mRNA, Fig. 8C, F $(3,61)=$ $185.1, P<0.0001$ ) in JX -stimulated cells clearly decreased. Alternatively, the IFN- $\beta$ upstream factors (RIGI, and TBK1) and IFN- $\beta$ downstream effectors (MX1 and ISG15) were also significantly down-regulated 
(Fig. 6). These results further confirmed that $P G R M C 1$ knockout promoted virus replication by efficiently suppressing RIG-I-mediated IFN- $\beta$ production.

\section{PGRMC1 knockout antagonized RIG-I ubiquitination}

RIG-I exists in an inactive closed conformation under normal conditions. RIG activation requires ubiquitination induced by the tripartite motif 25 (TRIM25) ubiquitin E3 ligase. Hence, we next addressed whether PGRMC1 knockout specifically suppresses RIG-I ubiquitination [28]. Toward this, we assessed RIG-I ubiquitination in PGRMC1-KO U251 cells or wild-type U251 cells transfected with pCAGGS-HA-Ub vector or the empty vector along with or without p3. Flag-RIG-I (Fig. 9), followed by infection with JX. The results of IP experiments clearly indicated that $P G R M C 1$ knockout markedly inhibited RIG-I ubiquitination. These results collectively revealed that the PGRMC1 knockout inhibited RIG-I ubiquitination in U251 cells, thereby suppressing RIG-I signal transduction.

\section{Discussion}

IAV is known to target the respiratory system, while some particular subtypes of IAV can infect the brain and cause serious damage to the CNS. Multiple routes of IAV invasion into the CNS after intranasal exposure have been reported. In this study, we isolated a strain of HPAIV H5N6 virus from duck, which showed high fatality and pathogenicity to mice. Some infected mice developed typical neurological symptoms such as balancing problems, hind limb weakness, and paralysis. Surprisingly, although the mouse survived till the completion of the experiment, hind limb paralysis did not improve. This observation was in accordance with the results of Hosseini et al., who reported that neuroinflammation caused by non-neurotropic and neurotropic IAV induces long-lasting impairments in hippocampal neuronal morphology and synaptic properties and cognitive function in adult animals [29]. The viral loads in the brain of mice in the infected group were dected examined using a $\mathrm{TCID}_{50}$ assay. These results confirmed that the $\mathrm{JX}$ virus can infect, replicate, and spread throughout the CNS. This agrees with the observation that viruses of the HPAIV H5 subtype are more frequently associated with CNS disease in humans [30].

The molecular mechanisms underlying the involvement of host factors in IAV infection in CNS remain obscure. Here, we identified 240 genes in the IAV-infected mice brains using a RNA-seq screen. These genes were involved in herpes simplex infection, glutamatergic synapse, and chemokine signaling pathway. Among these identified genes, H2-T23, H2-T24, IfI I, IIf7, and Stat2 were involved in other virus infections, such as those caused by the chikungunya virus, Duvenhage virus, and Zika virus. Zhao and co-workers observed that five virus-related proteins (TGTP, IFIT3, IFIT3-L, LCP1-1, and LCP1-2) were upregulated within $1 \mathrm{dpi}$ in the lethal virus-inoculated group, but did not show appreciable up-regulation until $3 \mathrm{dpi}$ in the non-lethal group. Their results suggest that proteomic approaches can distinguish the lethality of the influenza strain in a mouse model within $24 \mathrm{~h}$ of infection [31]. Interestingly, the interferonresponsive gene, Ifit3, which is reported to be involved in cognitive decline in aged mice [32], was 
specifically up-regulated in the hippocampus of H7N7-infected mice and is mainly expressed in granule cells (Cho et al., [33]).

PGRMC1 participates in cellular processes relevant to CNS diseases, including neuroprotection, axonal migration, and mitochondrial protection. Furthermore, it is highly expressed in the brain. However, the relationship between PGRMC1 and virus infection in the brain remains uninvestigated. Here, we observed that HPAIV H5N6 significantly inhibited PGRMC1 expression in the brain. This phenomenon has further been demonstrated in SK-N-SH and U251 Cells. Next, we investigated the effect of PGRMC1 on H5N6 and observed that AG205-mediated inhibition of PGRMC1 increased viral titers in these two cell lines. Although AG205 was advantageous for the proliferation of H5N6 in SK-N-SH and U251 cells, it promoted viral replication more in U251 cells than in SK-N-SH cells. U251 cells are derived from glial cells, which play a key role in regulating the immune responses in CNS. In addition, one of the first lines of host immunological defense against viruses involves the IFNs. Therefore, we hypothesized that AG205 treatment or $P G R M C 1$ knockout may inhibit the H5N6-mediated IFN signaling pathway. As expected, AG205 treatment or PGRMC1 knockout dramatically inhibited the type I interferon response, implying that PGRMC1 is a host factor restricting IAV JX infection in the CNS.

IAV genomes consist of eight negative sense single-stranded RNA segments (vRNA) that encode at least 14 viral proteins [34]. Hence, we aimed to identify the gene(s) involved in PGRMC1 inhibition. To this end, eight viral gene segments were individually cloned in the pHW2000 expression vector. However unfortunately, we observed that no single IAV JX gene segment was able to significantly down-regulate PGRMC1 (data not shown). This indicated that a single gene or protein is not sufficient for inhibiting PGRMC1 and that down-regulation of PGRMC1 is relevant for the biological characteristics of H5N6 virus.

During coevolution with hosts, IAV has developed multiple strategies to escape the host antiviral innate immunity, especially via blockade of the IFN system. Several IAV proteins have been well characterized as IFN antagonists, which antagonize the host IFN response at different steps. IAV antagonizes IFN response via various strategies, which involve viral proteins and host factors. For example, NS1 of IAV inhibits the activation of IRF3 and IFN- $\beta$ transcription. In addition, PB2 and PB1-F2 interact with MAVS, which repress IFN- $\beta$ expression [9]. In this study, H5N6 down-regulated PGRMC1 in the brain, which strongly suppressed the activation of the RIG-I-mediated IFN- $\beta$ signaling pathway. Furthermore, AG205 treatment or PGRMC1 knockout inhibited not only RIG-I expression stimulated by the H5N6 virus, but also RIG-I ubiquitination. These results suggest that IAV-induced down-regulation of PGRMC1 is involved in controlling RIG-I signaling, which is beneficial for the virus infection in the CNS. However, the mechanisms via which down-regulated PGRMC1 inhibits the expression and ubiquitination of RIG-I remain unclear. Possibly, down-regulated PGRMC1 perturbs the interaction between RIG-I and its E3 ligase, TRIM25, which warrants further investigations. Previous studies have assumed that PGRMC1 can alter its sub-cellular location under certain circumstances. Peluso suggested that as a transcription factor, PGRMC1 is involved in the regulation of gene transcription [35]. 
Microglia and astrocytes are the CNS resident cells most prominently involved in early innate responses to injury, autoimmune attack, or infection [27]. Unfortunately, pathogens can induce devastating inflammatory damage to the CNS, leading to rapid mortality or long-term neurological disabilities. Nevertheless, some viruses may survive as latent infections in conducive environments, provided they become dormant and do not proliferate. Thus, the CNS 'protects' these pathogens.

Further studies are necessary to more precisely explore the specific mechanism in this study. First, the mechanism of $\mathrm{H} 5 \mathrm{~N} 6$ virus downregulating PGRMC1 express still need to be intensively studied. In addition, this mechanism has not been validated in vivo. so further studies will need to be conducted on PGRMC1 knockout mice.

\section{Conclusions}

In summary, we identified PGRMC1 as a novel host factor involved in the replication and propagation of IAV H5N6 in the host brain. We also observed that PGRMC1 antagonism was required for evasion from host immune response during IAV infection via inhibition of the expression and ubiquitination of RIG-I, thereby blocking activation RIG-I-mediated IFN- $\beta$ signaling pathway and downstream antiviral gene expression. Overall, our study revealed a newly identified regulatory mechanism used by IAV H5N6 to ensure its life cycle in the CNS.

\section{Abbreviations}

IAV influenza A virus

CNS central nervous system

PGRMC1 progesterone receptor membrane component-1

RIG-I retinoic acid-inducible gene I

HPAIV highly pathogenic avian influenza virus

PRRs pattern recognition receptors

MAVS mitochondrial antiviral signaling

MDA-5 melanoma differentiation-associated gene- 5

TBK-1 TANK binding kinase 1

IRF3 FN regulatory factor 3

CRISPR clustered regularly interspaced short palindromic repeats 
co-IP co-immunoprecipitation

TCID50 50\% tissue culture infective dose

MOI multiplicity of infection

MX1 myxovirus resistance protein 1

ISG15 interferon-stimulated gene 15

\section{Declarations}

\section{Acknowledgments}

We thank LC-Bio Corporation for assistance with RNA sequencing and gene expression analysis. We also thank professor Zhengfan Jiang from Peking University providing the luciferase reporter constructs including IFNB promoter (IFNB-luc).

\section{Ethics approval}

All animal experiments were approved by the Research Ethics Committee, Huazhong Agricultural University, Hubei, China (HZAUMO-2016-022) and were performed in accordance with the Guidelines for the Care and Use of Laboratory Animals of the Research Ethics Committee, Huazhong Agricultural University, Hubei, China.

\section{Funding}

This research was supported by the National Key Research and Development Program of China (2016YFD0500205) and National Natural Science Foundation of China (31702255 and 31820103015).

\section{Availability of data and materials}

All data and materials are available upon request.

\section{Authors' contributions}

$\mathrm{KH}$ performed the majority of experiments and statistical analysis and wrote the manuscript. YFZ, WG, YY, LLJ, YIY, YMW, CFL, XLH, XMS participated in the protocol execution and data collection. KH, ZZ, YFZ, LZZ and MLJ provided the critical review of the manuscript. MLJ was responsible for editing and revising the manuscript for the final version. All authors have read and approved the final version of the manuscript.

\section{Competing interests}

No potential conflict of interest was reported by the authors. 


\section{Consent for publication}

Not applicable

\section{References}

1. Jiao P, Song H, Liu X, Song Y, Cui J, Wu S, Ye J, Qu N, Zhang T, Liao M: Pathogenicity, Transmission and Antigenic Variation of H5N1 Highly Pathogenic Avian Influenza Viruses. Front Microbio/ 2016, 7:635.

2. Ding X, Luo J, Quan L, Wu A, Jiang T: Evolutionary genotypes of influenza A (H7N9) viruses over five epidemic waves in China. Infect Genet Evol 2017, 55:269-276.

3. Bi Y, Chen Q, Wang Q, Chen J, Jin T, Wong G, Quan C, Liu J, Wu J, Yin R, et al: Genesis, Evolution and Prevalence of H5N6 Avian Influenza Viruses in China. Cell Host Microbe 2016, 20:810-821.

4. You F, Sun H, Zhou X, Sun W, Liang S, Zhai Z, Jiang Z: PCBP2 mediates degradation of the adaptor MAVS via the HECT ubiquitin ligase AIP4. Nat Immunol 2009, 10:1300-1308.

5. Iwasaki A, Pillai PS: Innate immunity to influenza virus infection. Nat Rev Immunol 2014, 14:315-328.

6. Tavares LP, Teixeira MM, Garcia CC: The inflammatory response triggered by Influenza virus: a two edged sword. Inflamm Res 2017, 66:283-302.

7. Shah M, Anwar MA, Kim JH, Choi S: Advances in Antiviral Therapies Targeting Toll-like Receptors. Expert Opin Investig Drugs 2016, 25:437-453.

8. Rajsbaum R, Albrecht RA, Wang MK, Maharaj NP, Versteeg GA, Nistal-Villan E, Garcia-Sastre A, Gack MU: Species-specific inhibition of RIG-I ubiquitination and IFN induction by the influenza A virus NS1 protein. PLoS Pathog 2012, 8:e1003059.

9. Varga ZT, Ramos I, Hai R, Schmolke M, Garcia-Sastre A, Fernandez-Sesma A, Palese P: The influenza virus protein PB1-F2 inhibits the induction of type I interferon at the level of the MAVS adaptor protein. PLoS Pathog 2011, 7:e1002067.

10. Short KR, Veeris R, Leijten LM, van den Brand JM, Jong VL, Stittelaar K, Osterhaus A, Andeweg A, van Riel D: Proinflammatory Cytokine Responses in Extra-Respiratory Tissues During Severe Influenza. $J$ Infect Dis 2017, 216:829-833.

11. Toovey S: Influenza-associated central nervous system dysfunction: a literature review. Travel Med Infect Dis 2008, 6:114-124.

12. Holtenius J, Gillman A: The Spanish flu in Uppsala, clinical and epidemiological impact of the influenza pandemic 1918-1919 on a Swedish county. Infect Ecol Epidemiol 2014, 4.

13. Sheng ZM, Chertow DS, Ambroggio X, McCall S, Przygodzki RM, Cunningham RE, Maximova OA, Kash JC, Morens DM, Taubenberger JK: Autopsy series of 68 cases dying before and during the 1918 influenza pandemic peak. Proc Natl Acad Sci U S A 2011, 108:16416-16421.

14. Korteweg C, Gu J: Pathology, molecular biology, and pathogenesis of avian influenza A (H5N1) infection in humans. Am J Pathol 2008, 172:1155-1170. 
15. Siegers JY, van de Bildt MWG, Lin Z, Leijten LM, Lavrijssen RAM, Bestebroer T, Spronken MIJ, De Zeeuw Cl, Gao Z, Schrauwen EJA, et al: Viral Factors Important for Efficient Replication of Influenza A Viruses in Cells of the Central Nervous System. J Virol 2019, 93.

16. Leyva-Grado VH, Churchill L, Harding J, Krueger JM: The olfactory nerve has a role in the body temperature and brain cytokine responses to influenza virus. Brain Behav Immun 2010, 24:281-288.

17. Kuiken T, Taubenberger JK: Pathology of human influenza revisited. Vaccine 2008, 26 Suppl 4:D5966.

18. Mifsud W, Bateman A: Membrane-bound progesterone receptors contain a cytochrome b5-like ligandbinding domain. Genome Biol 2002, 3:RESEARCH0068.

19. Hand RA, Craven RJ: Hpr6.6 protein mediates cell death from oxidative damage in MCF-7 human breast cancer cells. J Cell Biochem 2003, 90:534-547.

20. Teakel SL, Ludescher M, Thejer BM, Poschmann G, Forwood JK, Neubauer H, Cahill MA: Protein complexes including PGRMC1 and actin-associated proteins are disrupted by AG-205. Biochem Biophys Res Commun 2020, 524:64-69.

21. Nolte I, Jeckel D, Wieland FT, Sohn K: Localization and topology of ratp28, a member of a novel family of putative steroid-binding proteins. Biochim Biophys Acta 2000, 1543:123-130.

22. Beausoleil SA, Jedrychowski M, Schwartz D, Elias JE, Villen J, Li J, Cohn MA, Cantley LC, Gygi SP: Large-scale characterization of HeLa cell nuclear phosphoproteins. Proc Natl Acad Sci U S A 2004, 101:12130-12135.

23. Kimura I, Yoshioka M, Konishi M, Miyake A, Itoh N: Neudesin, a novel secreted protein with a unique primary structure and neurotrophic activity. J Neurosci Res 2005, 79:287-294.

24. Kimura I, Konishi M, Miyake A, Fujimoto M, Itoh N: Neudesin, a secreted factor, promotes neural cell proliferation and neuronal differentiation in mouse neural precursor cells. J Neurosci Res 2006, 83:1415-1424.

25. Ramakrishnan MA: Determination of $50 \%$ endpoint titer using a simple formula. World J Virol 2016 , 5:85-86.

26. Zhao L, Zhang X, Wu Z, Huang K, Sun X, Chen H, Jin M: The Downregulation of MicroRNA hsa-miR340-5p in IAV-Infected A549 Cells Suppresses Viral Replication by Targeting RIG-I and OAS2. Mol Ther Nucleic Acids 2019, 14:509-519.

27. Biswas S, Cottarelli A, Agalliu D: Neuronal and glial regulation of CNS angiogenesis and barriergenesis. Development 2020, 147.

28. Gack MU, Shin YC, Joo CH, Urano T, Liang C, Sun L, Takeuchi O, Akira S, Chen Z, Inoue S, Jung JU: TRIM25 RING-finger E3 ubiquitin ligase is essential for RIG-I-mediated antiviral activity. Nature 2007, 446:916-920.

29. Hosseini S, Wilk E, Michaelsen-Preusse K, Gerhauser I, Baumgartner W, Geffers R, Schughart K, Korte M: Long-Term Neuroinflammation Induced by Influenza A Virus Infection and the Impact on Hippocampal Neuron Morphology and Function. J Neurosci 2018, 38:3060-3080. 
30. Gu J, Xie Z, Gao Z, Liu J, Korteweg C, Ye J, Lau LT, Lu J, Gao Z, Zhang B, et al: H5N1 infection of the respiratory tract and beyond: a molecular pathology study. Lancet 2007, 370:1137-1145.

31. Zhao D, Liang L, Li Y, Liu L, Guan Y, Jiang Y, Chen H: Proteomic analysis of the lungs of mice infected with different pathotypes of H5N1 avian influenza viruses. Proteomics 2012, 12:1970-1982.

32. Bordner KA, Kitchen RR, Carlyle B, George ED, Mahajan MC, Mane SM, Taylor JR, Simen AA: Parallel declines in cognition, motivation, and locomotion in aging mice: association with immune gene upregulation in the medial prefrontal cortex. Exp Geronto/ 2011, 46:643-659.

33. Cho H, Proll SC, Szretter KJ, Katze MG, Gale M, Jr., Diamond MS: Differential innate immune response programs in neuronal subtypes determine susceptibility to infection in the brain by positivestranded RNA viruses. Nat Med 2013, 19:458-464.

34. Ampomah PB, Lim LHK: Influenza A virus-induced apoptosis and virus propagation. Apoptosis 2020, 25:1-11.

35. Terzaghi L, Luciano AM, Dall'Acqua PC, Modina SC, Peluso JJ, Lodde V: PGRMC1 localization and putative function in the nucleolus of bovine granulosa cells and oocytes. Reproduction 2018, 155:273-282.

\section{Figures}




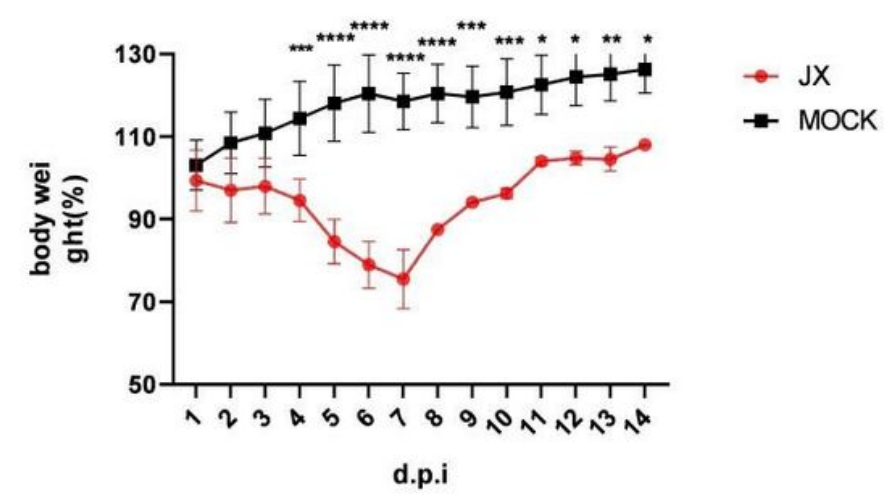

C

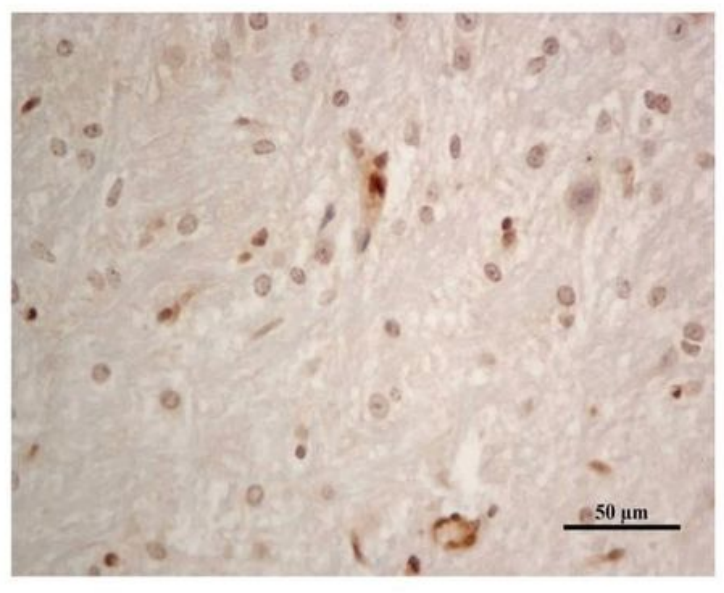

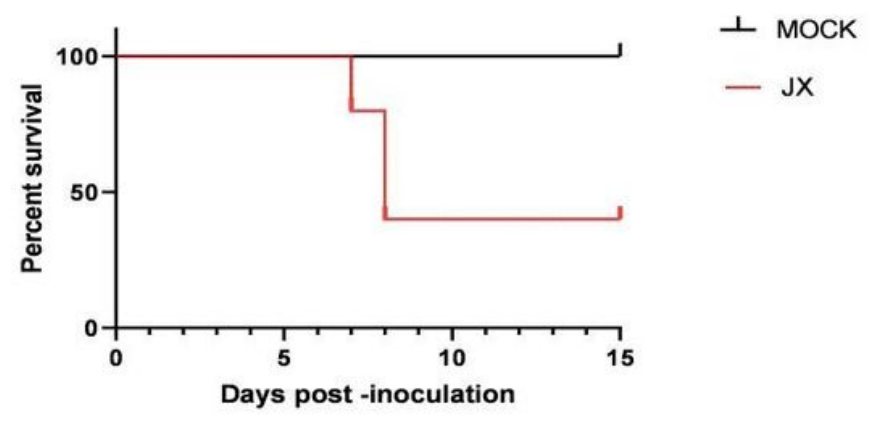

D

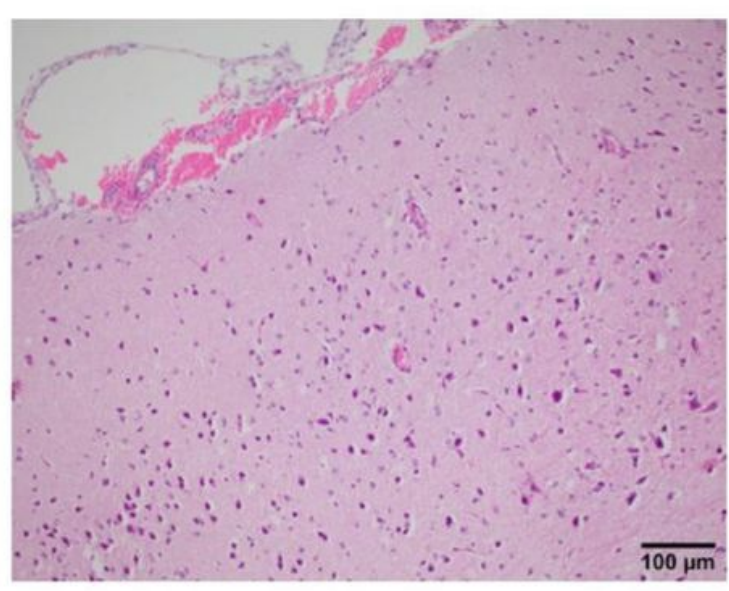

Figure 1

Infection of BALB/c mice $(n=11)$ with H5N6 virus. Mice receiving PBS were used as controls. The mice were monitored for 14 days. (A) Body weight changes depicted as percentage of the starting weight of mice ( ${ }^{*}, \mathrm{P}<0.05 ; * \star, \mathrm{P}<0.01 ; * \star \star, \mathrm{P}<0.001 ; * \star \star \star, \mathrm{P}<0.0001$, using two-way ANOVA). (B) Survival of infected mice (Mantel-Cox log-rank test). (C) Immunohistochemical analysis of the nucleoprotein antigen in brainstem . (D) Degeneration and necrosis of neurons and local glial cell nodules in the cerebral cortex. 
A

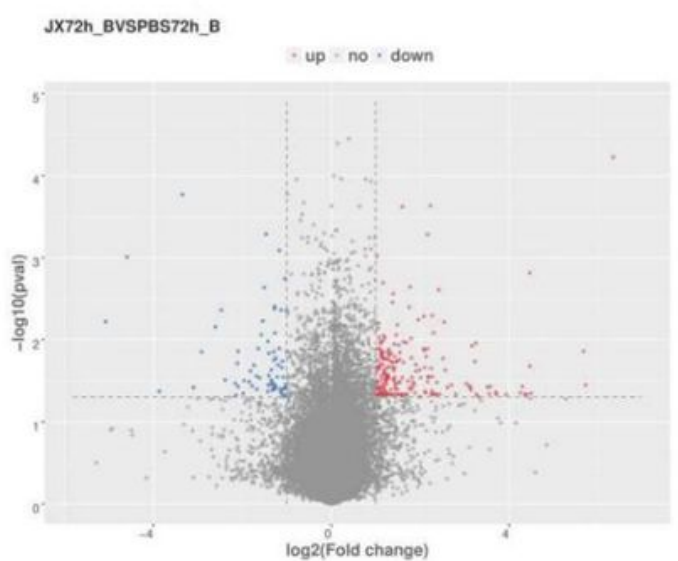

C

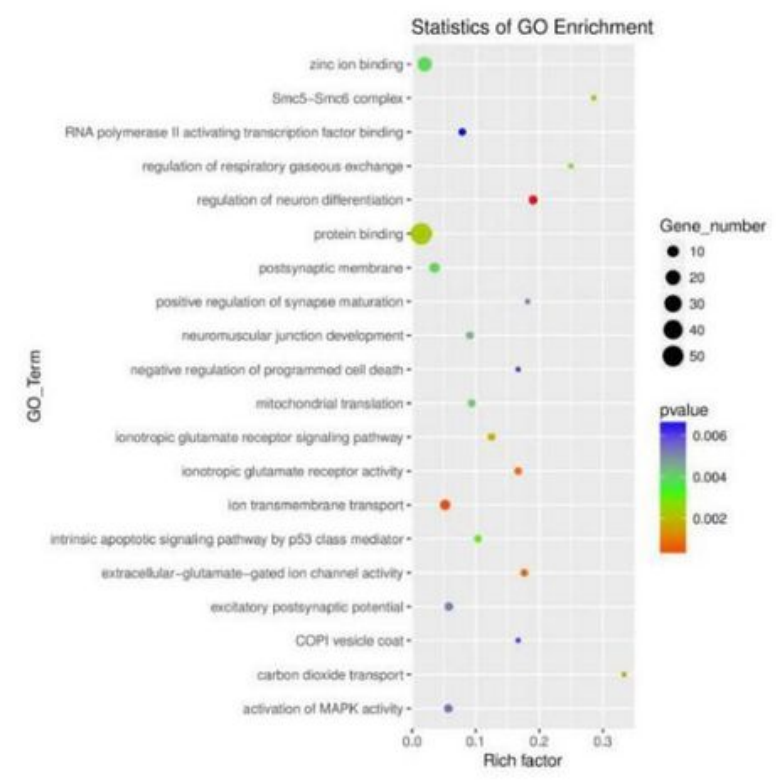

B

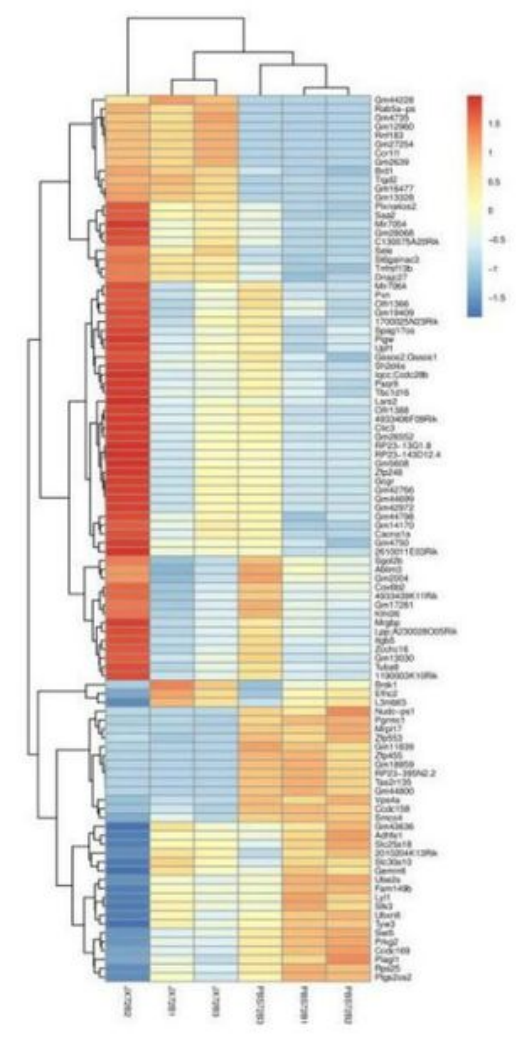

D

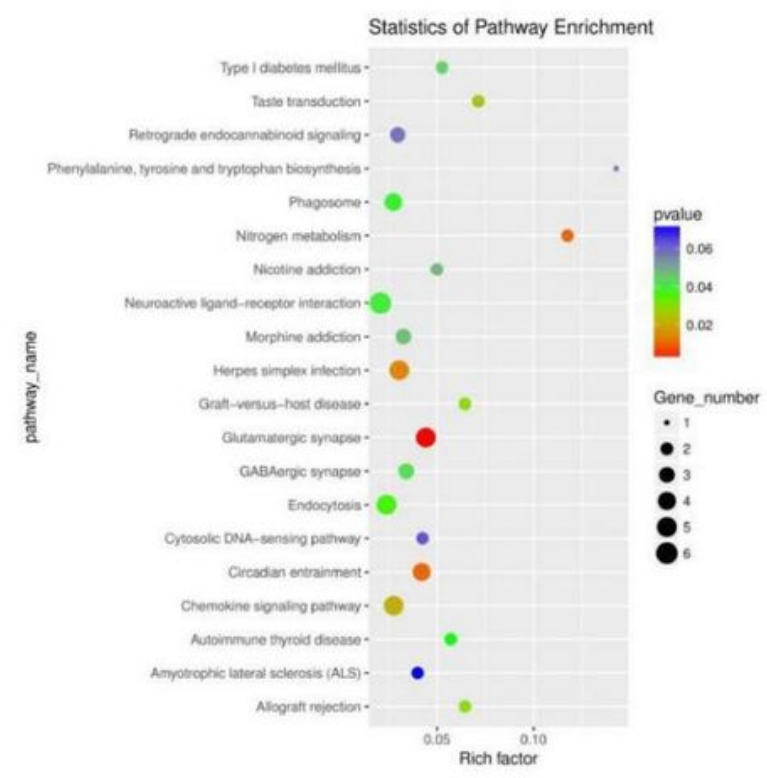

\section{Figure 2}

Analysis of the transcriptome profiles of brain tissue after infection with H5N6 virus. (A) Gene volcano plot and (B) heat map. (C) Scatter plot showing Gene Ontology (GO) and Kyoto Encyclopedia of Genes and Genomes (KEGG) enrichment analysis. 
A

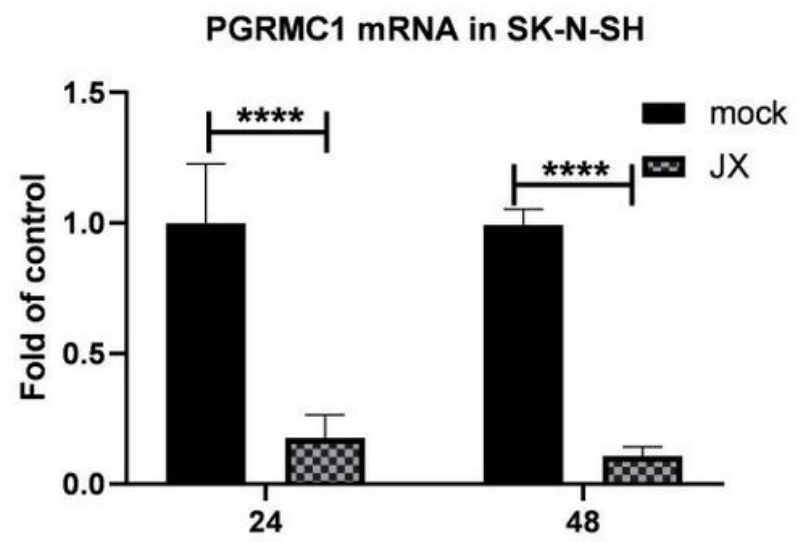

Hours post-infection
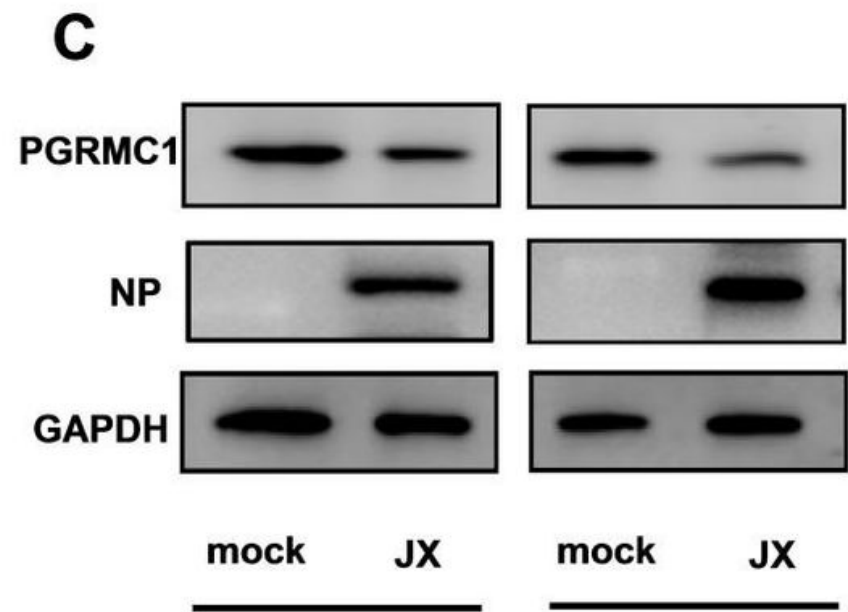

24h

\section{B}

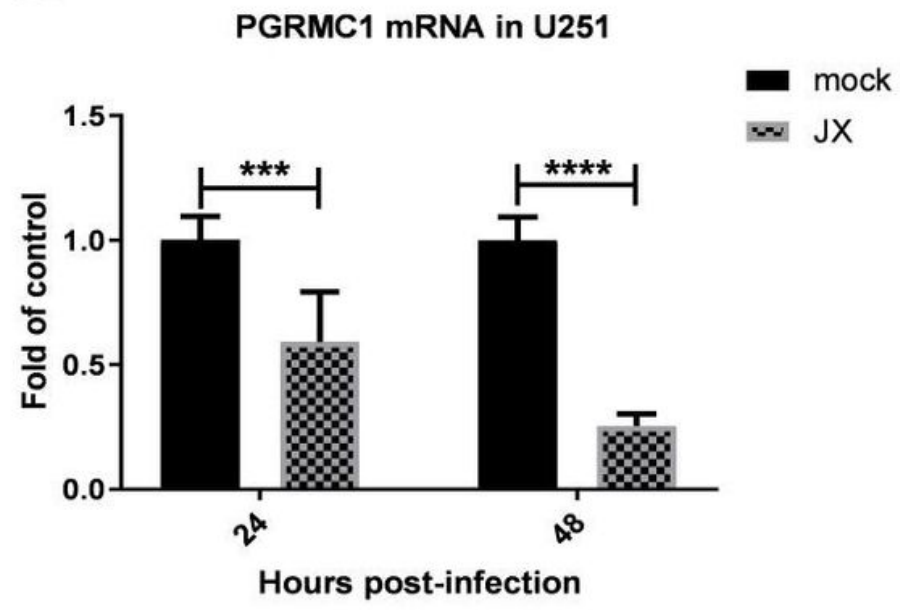

D

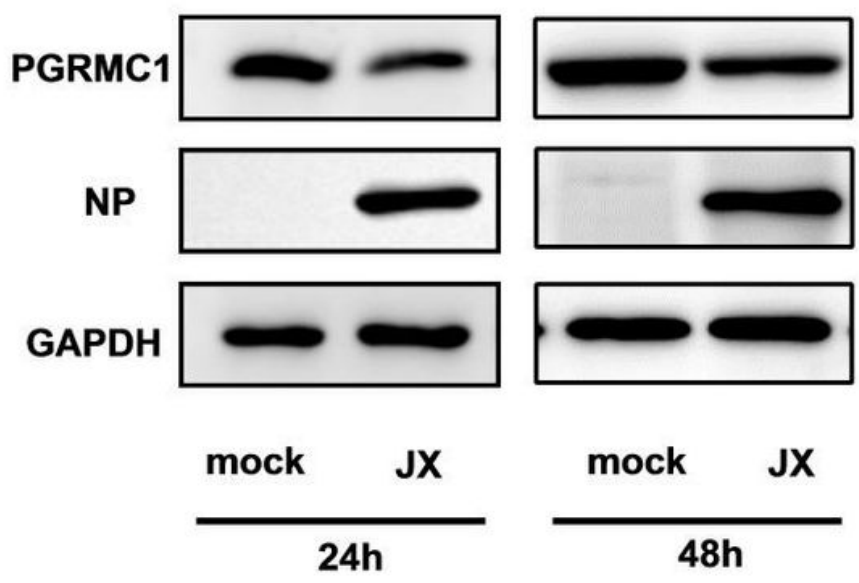

Figure 3

JX inhibited PGRMC1 expression in vitro. PGRMC1 expression level in SK-N-SH cells (A and C) and U251 cells(B and D). Cells were infected with JX at an MOI of 0.01. Samples were collected at 24 and $48 \mathrm{hpi}$, followed by qRT-PCR and western blotting to determine the mRNA ( $A$ and $B$ ) and protein ( $C$ and $D$ ) levels of PGRMC1, respectively. For real-time PCR analysis, the mRNA level was normalized to the GAPDH level. The data are presented as the mean $\pm S D$ from three independent experiments $(*, P<0.05 ; * \star, P<0.01$; *ᄎ*, $\mathrm{P}<0.001 ; * \star \star \star, \mathrm{P}<0.0001$, using two-way ANOVA). GAPDH was used as a loading control for western blot analysis. 
A

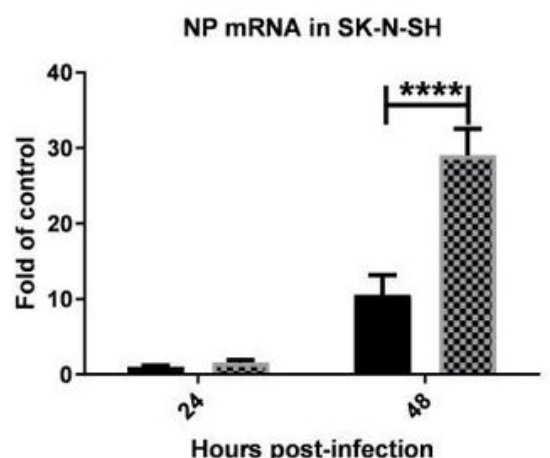

C

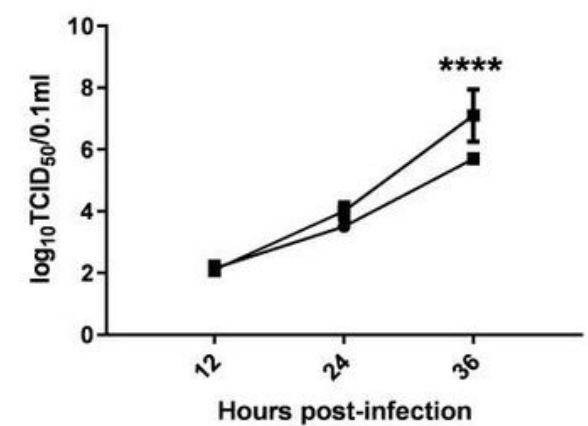

E

Viral titers in U251

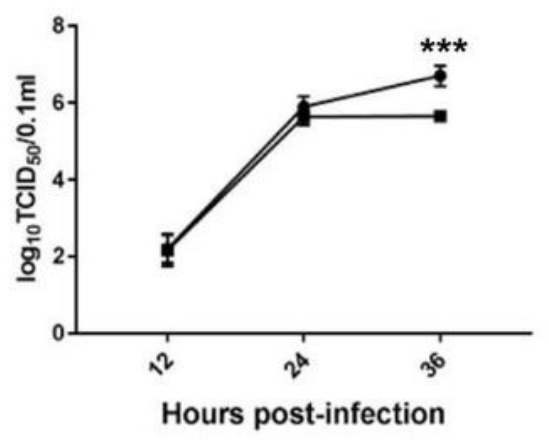

B

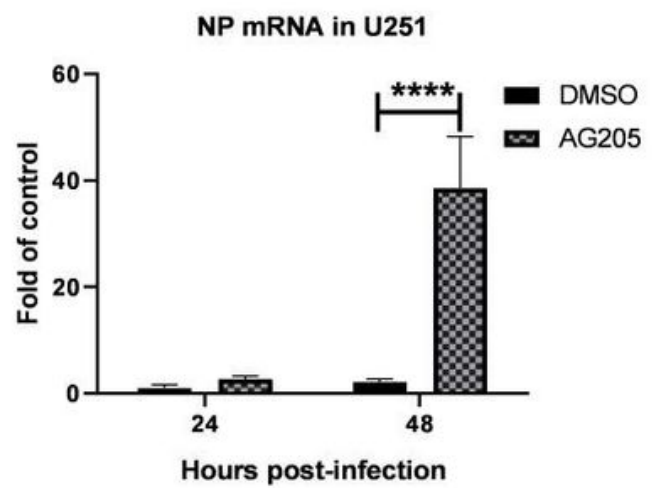

D

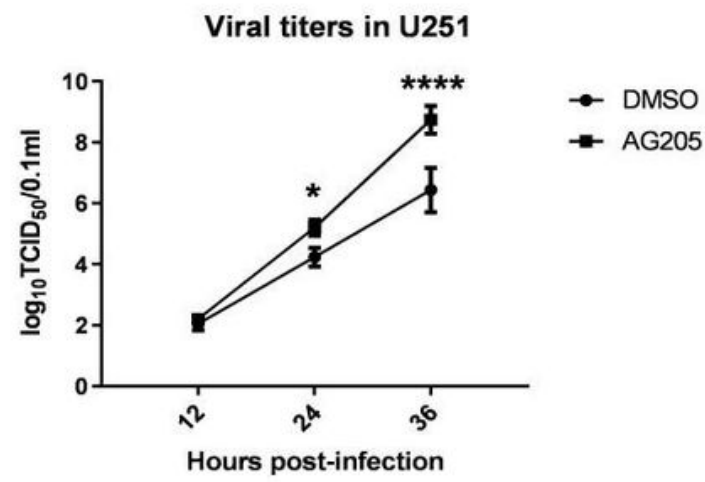

F

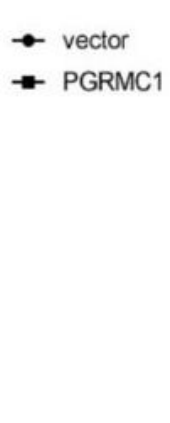

PGRMC1

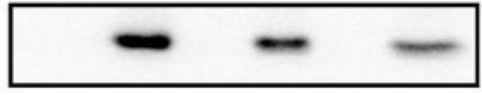

GAPDH

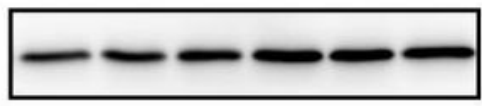

vector

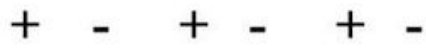

PGRMC1

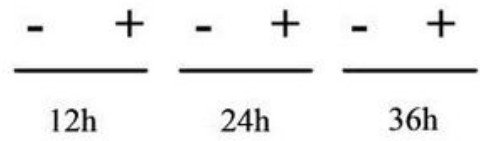

Figure 4

Specific inhibition of PGRMC1 by AG205 increased JX H5N6 virus replication. SK-N-SH cells (A and C) and $\mathrm{U} 251$ cells (B and D) were pretreated with $15 \mu \mathrm{M}$ AG205 or DMSO over $24 \mathrm{~h}$. Cells were infected with $\mathrm{JX}$ at an MOI of 0.01 . Samples were collected at 12, 24, 36 and $48 \mathrm{hpi}$, viral NP mRNA and viral titers were determined using qRT-PCR and TCID 50, respectively. U251 cells (E and F) were transfected with FlagPGRMC1 and empty vectors. After $24 \mathrm{~h}$, the cells were infected with JX at MOI of 0.01 and expression 
was detected using western blotting. TCID50 assays (E) for the virus titer of JX were performed to estimate virus multiplication at 12, 24, and 36 h.p.i. Overexpression of PGRMC1 in U251 cells was detected by western blot $(F)$. The data are presented as the mean \pm SD from three independent experiments ( $*, \mathrm{P}<0.05 ; * *, \mathrm{P}<0.01 ; * \star *, \mathrm{P}<0.001$; ****, $\mathrm{P}<0.0001$, using two-way ANOVA).

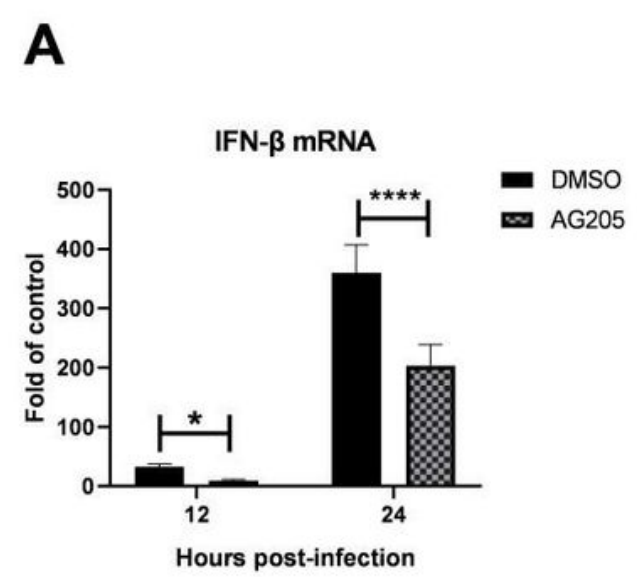

B

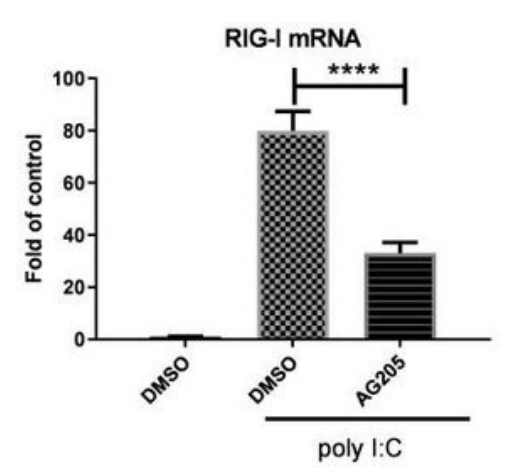

$\mathbf{E}$

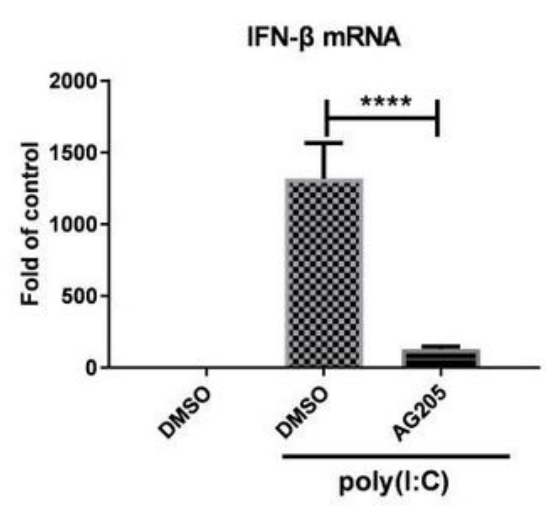

C

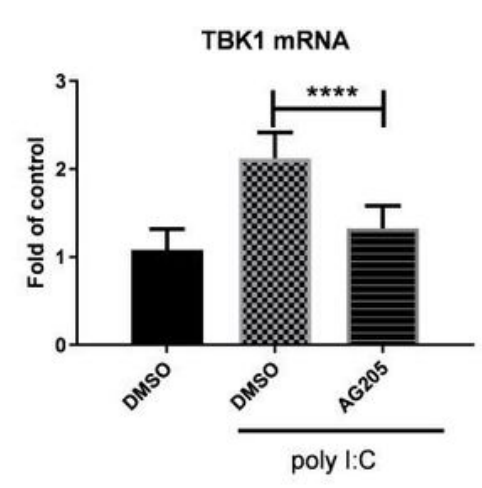

$\mathbf{F}$

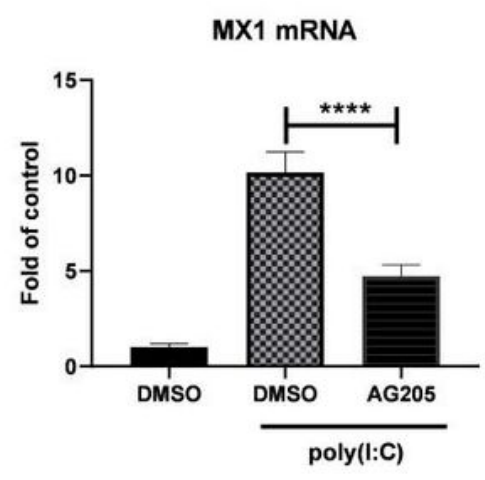

D

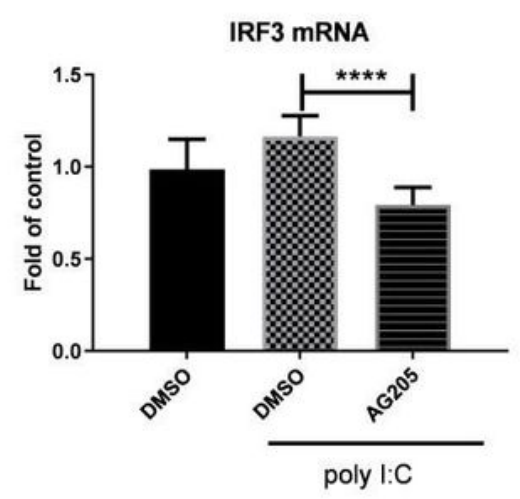

G

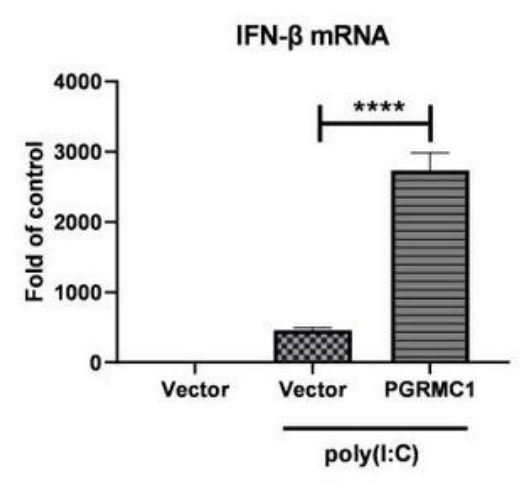

Figure 5 
AG205 negatively modulated the IFN- $\beta$-inducing pathway. U251 cells were pretreated with $15 \mu \mathrm{M}$ AG205 or DMSO for $24 \mathrm{~h}$. The cells were infected with $\mathrm{JX}$ at an MOI of 0.01 (A) or were stimulated with $100 \mathrm{ng}$ of poly $(\mathrm{l}: \mathrm{C})(\mathrm{B}-\mathrm{G})$ for $24 \mathrm{~h}$. The mRNA levels of the target genes were detected and normalized to the GAPDH level, and the expression in the DMSO and poly(l:C)-unstimulated groups were set to 1 . The data are presented as the mean \pm SD from three independent experiments $(*, P<0.05 ; * \star, P<0.01 ; * \star *, P<0.001$; $\star \star \star \star, P<0.0001$, A was analysed by repeated measures two-way ANOVA.. B-G were analysed by one-way ANOVA).

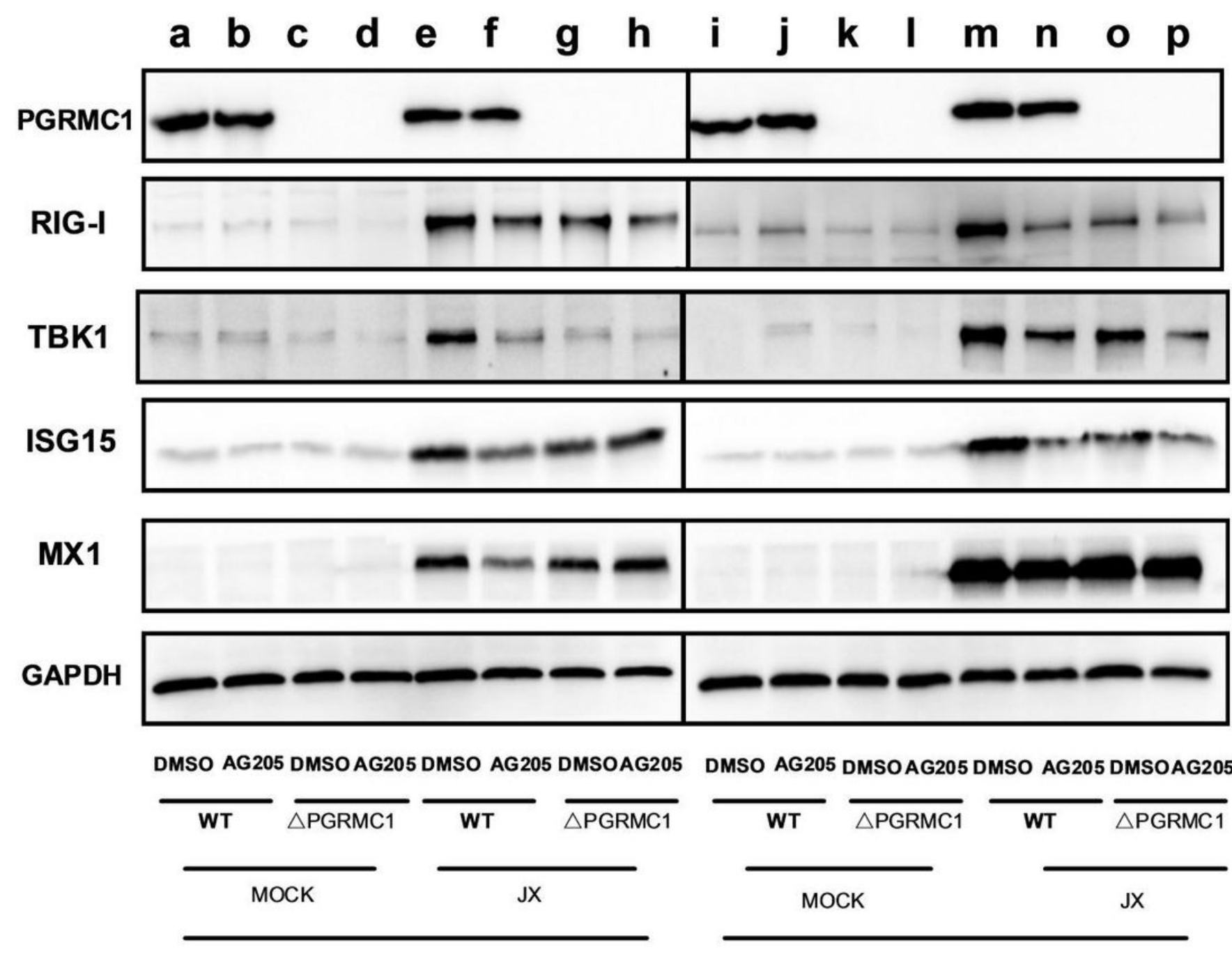

12 h.p.i

24 h.p.i

Figure 6

AG205 or PGRMC1 knockout inhibited the RIG-I-mediated IFN- $\beta$ signaling pathway. PGRMC1-KO U251 cells and wild type cells were treated with $15 \mu \mathrm{M}$ AG 205 or DMSO for $24 \mathrm{~h}$. Cells were infected with JX at $\mathrm{MOI}$ of 0.01 . Samples were collected at 12 and $24 \mathrm{~h}$. IFN- $\beta$ signaling molecules were detected using western blotting. 
A

B

C
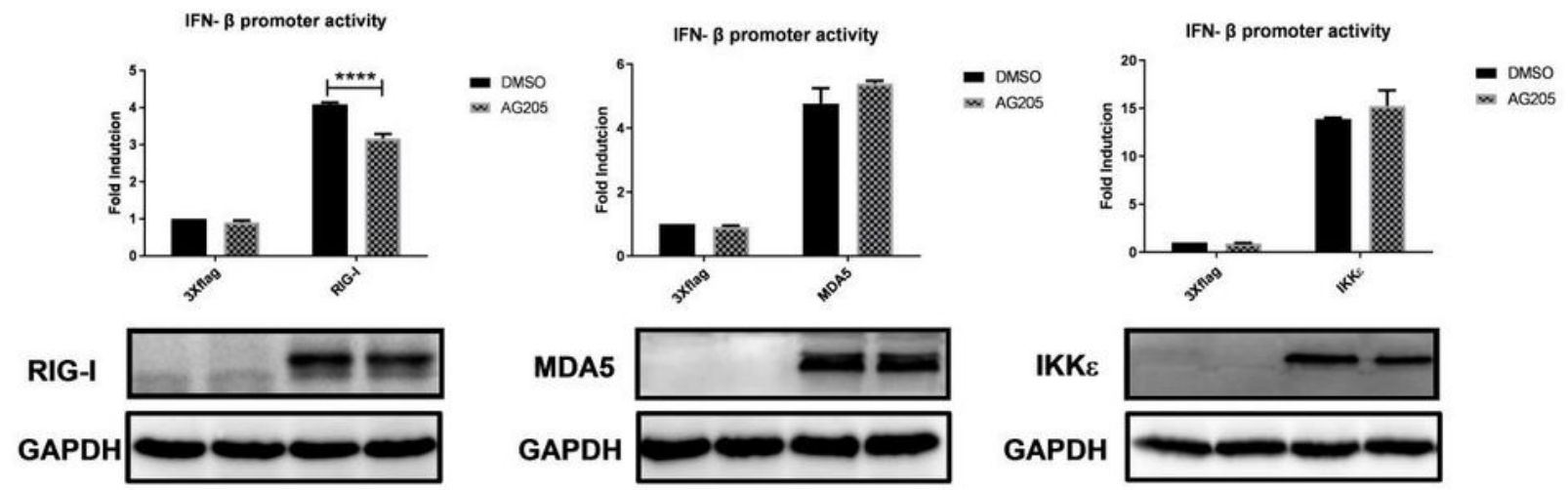

IKK $\varepsilon$

GAPDH

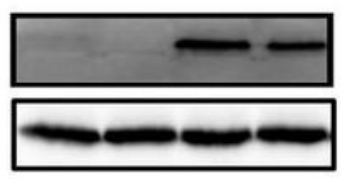

E

$\mathbf{F}$
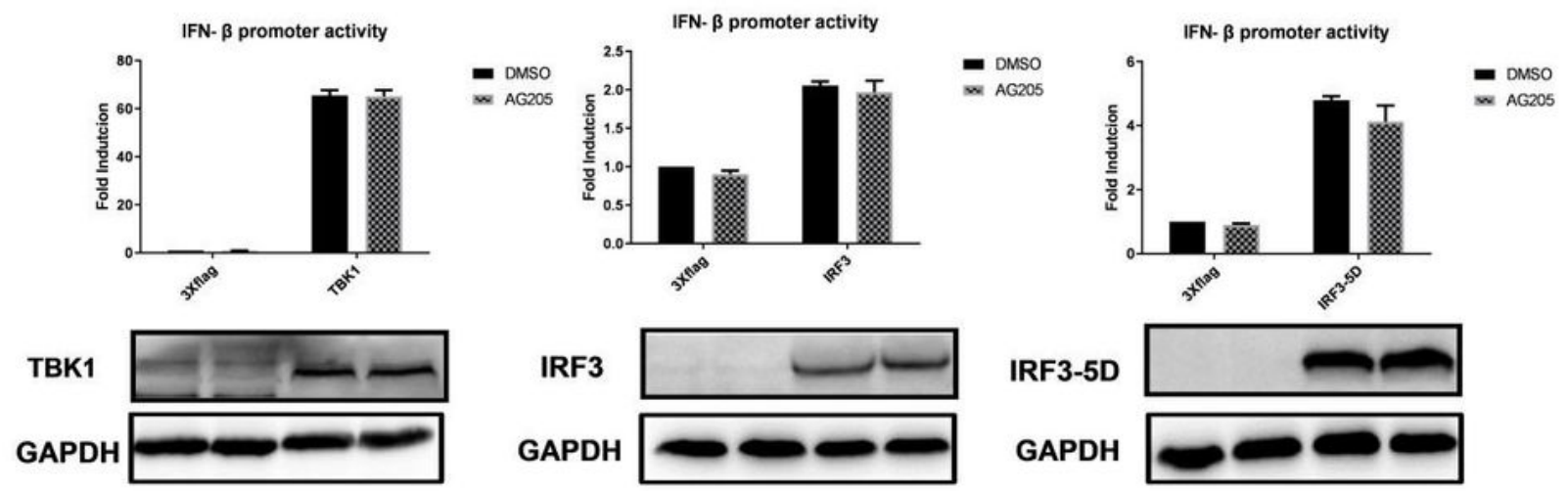

IRF3-5D

GAPDH

H
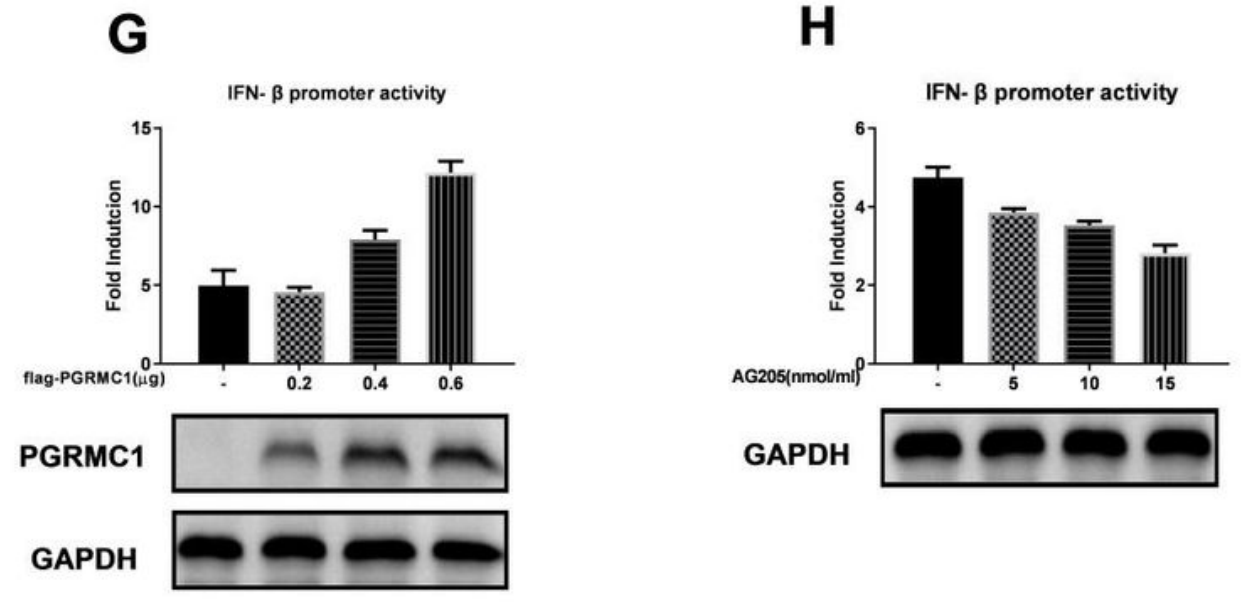

Figure 7

AG205 inhibited IFN- $\beta$ promoter activity induced by RIG-I-mediated IFN- $\beta$ signaling molecules. Effect of AG205 or PGRMC1 on the activation of IFN- $\beta$ promoter induced by RIG-I (A), MDA5 (B), IKKع (C) TBK1 (D), IRF3 (E), and IRF3-5D (F). HEK293T cells were treated with $15 \mu \mathrm{M}$ AG 205 or DMSO for $24 \mathrm{~h}$. Then, the cells were transfected with IFN- $\beta$-luc, pRL-TK, and the indicated expression plasmids of the signaling molecules. The effect of different doses of PGRMC1 (G) or AG205 (H) on the activation of IFN- $\beta$ promoter 
induced by RIG-I was measured $24 \mathrm{~h}$ post-transfection using the luciferase activity assay. The expression level of each signaling molecule was detected using western blotting with an anti-Flag antibody. The data are presented as the mean $\pm S D$ from three independent experiments $(*, P<0.05 ; * \star, P<0.01 ; * \star \star, P<$ $0.001 ; * \star \star \star, P<0.0001, A-F$ were analysed by repeated measures two-way ANOVA.. $G, H$ were analysed by one-way ANOVA).

A

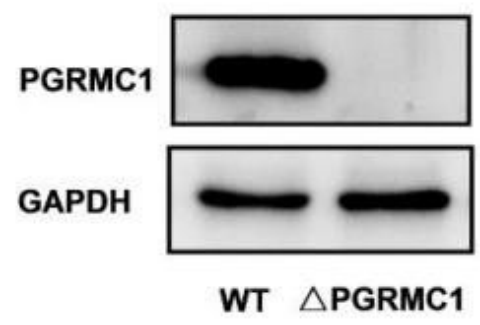

B

Viral titers in U251

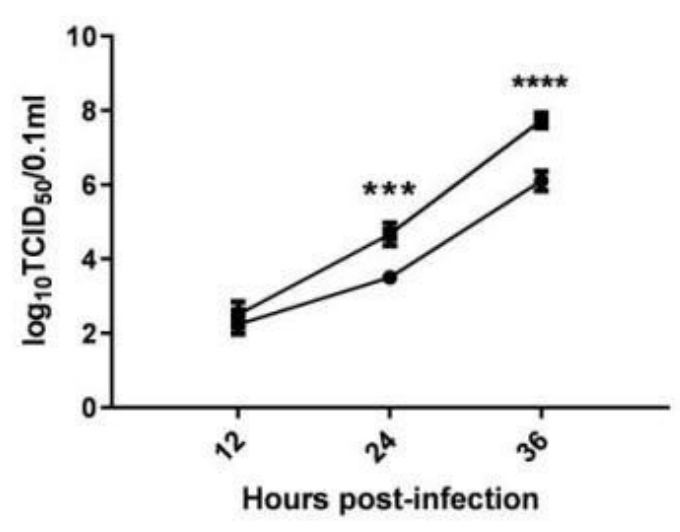

$\rightarrow$ WT-JX
$\rightarrow \triangle P G R M C 1-J X$

C

IFN- $\beta$ mRNA in U251

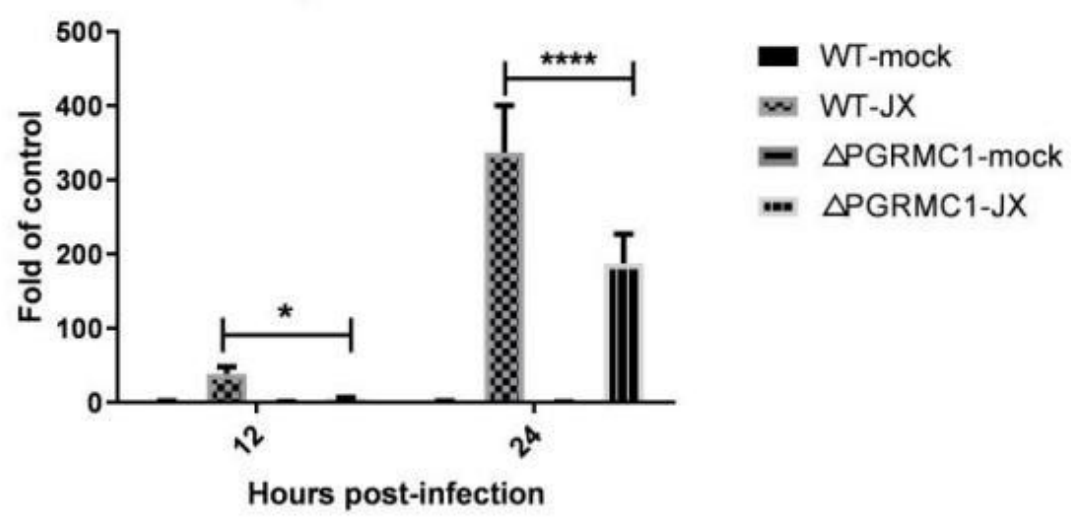

Figure 8 
Effect of PGRMC1 knockout on IAV replication and the RIG-I-mediated IFN- $\beta$ signaling pathway. Generation of PGRMC1-KO U251 cells using the CRISPR/Cas9 system. PGRMC1 knockout was confirmed using western blotting (A). PGRMC1-KO U251 cells ( $\triangle \mathrm{PGRMC1)}$ ) or wild-type U251 cells (WT) were infected with JX H5N6 virus at MOI of 0.01. Viral titers were determined using TCID50 on MDCK cells (B). The mRNA levels of the IFN- $\beta$ was detected and normalized to the GAPDH level (C) (means \pm SD from

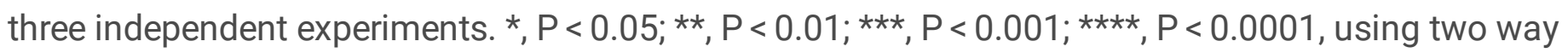
ANOVA).
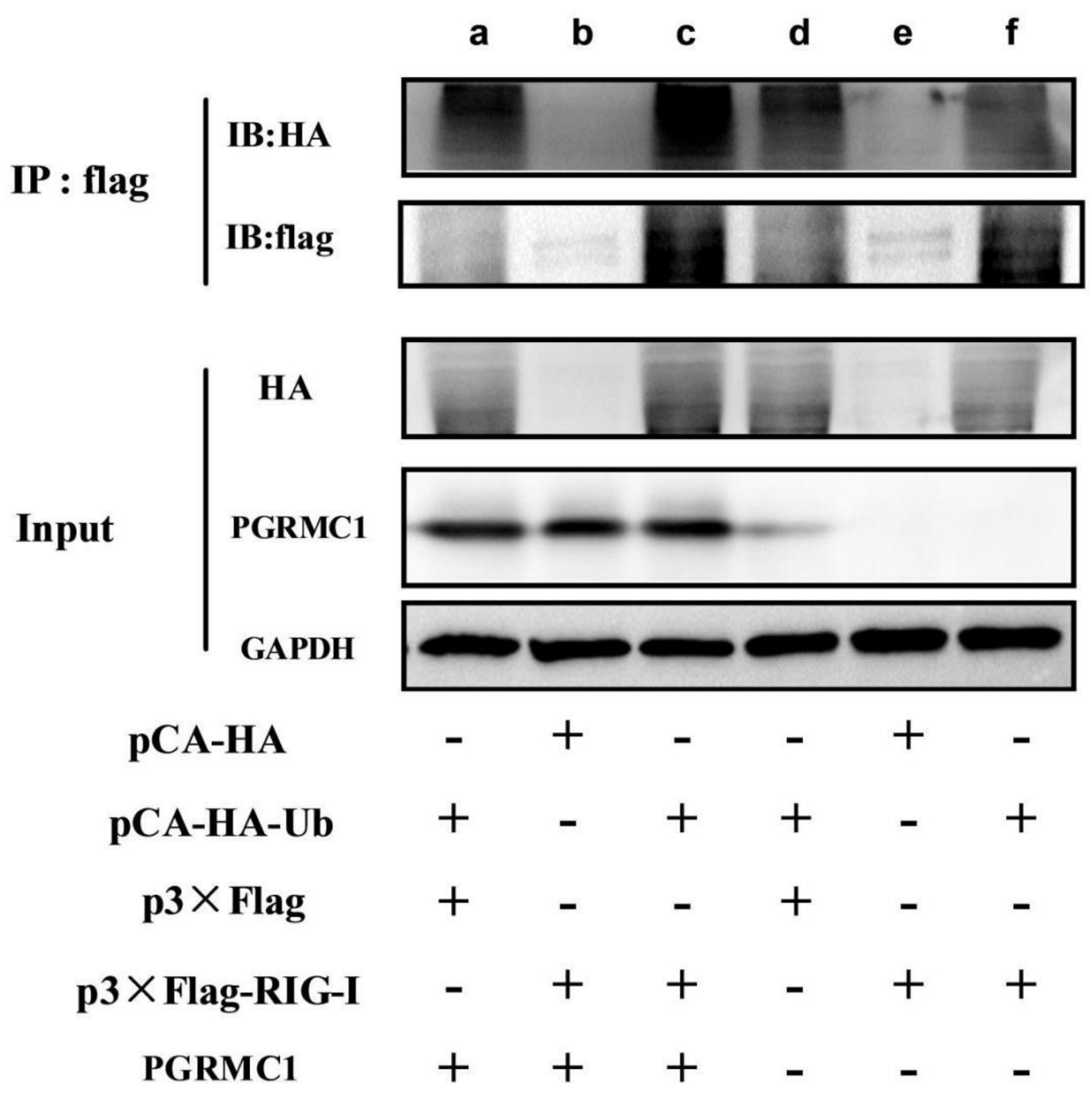

Figure 9 
PGRMC1 knockout inhibited RIG-I ubiquitination. PGRMC1-KO U251 cells and wild type cells were transfected with Flag-RIG-I and pUb-HA. After $24 \mathrm{~h}$, the cells were infected with JX at MOI of 0.01. After 24 $\mathrm{h}$, the cells were subjected to anti-Flag immunoprecipitation and immunoblotting with anti-HA to monitor RIG-I ubiquitination. 